\title{
Price vs Quantity in a Duopoly Supergame with Nash Punishments
}

\author{
Serena Baldelli ${ }^{\S}$ and Luca Lambertini ${ }^{\#}$ \\ Department of Economics, University of Bologna \\ Strada Maggiore 45, 40125 Bologna, Italy \\ Fax: +39-051-2092623; phone: +39-051-2092664 \\ $\S$ sere.net@libero.it; \# lamberti@spbo.unibo.it \\ June 9, 2004
}

\begin{abstract}
We examine the endogenous choice between price and quantity behaviour in a duopoly supergame with product differentiation. We find that (i) if cartel profits are evenly split between firms, then only symmetric equilibria obtains; (i) if instead the additional profits available through collusion are split according to the Nash bargaining solution, there are parameter regions where all subgame perfect equilibria are asymmetric, with firms colluding in price-quantity supergames.
\end{abstract}

JEL Classification: C72, D43, L13

Keywords: repeated games, collusion, product differentiation 


\section{Introduction}

A large body of literature has examined the relative stability of collusion in Bertrand and Cournot markets, parametrised over the degree of product differentiation. ${ }^{1}$ Relatively few, instead, allow for the possibility that firms be asymmetric in terms of their market variables, considering thus pricequantity supergames (Lambertini, 1997; Albæk and Lambertini, 2004). In Rothschild (1995) and Lambertini and Schultz (2001, 2003), the possibility that firms optimally choose whether to be price- or quantity-setters in each period is considered. Notwithstanding the fact that firms are allowed to select different market variables, from none of these contributions there emerge asymmetric cartels at the subgame perfect equilibria. Does this entail that antitrust agencies must not worry about such types of collusive behaviour? The underlying symmetry between firms, in terms of technology and product differentiation, that is a consistent feature of the models belonging to this stream of literature seems to provide an intuitive explanation for this result.

Moreover, neither Lambertini (1997) nor Albæk and Lambertini (2004) investigate which market variable yields the highest profit to the implicit cartel members for a given discount factor. Instead, they identify the lowest discount factor compatible with a subgame perfect equilibrium where firms stabilise collusion at the monopoly profits, being committed to set quantities or prices in all phases of the infinitely repeated duopoly game. Then, under the assumption that firms are able to collude along the frontier of industry profits irrespective of the market variable(s) being set, a meta-game is introduced, whereby firms choose market variables once and for all, the payoffs

\footnotetext{
${ }^{1}$ The number of contributions in this field is very large. See Deneckere $(1983,1984)$, Majerus (1988), Ross (1992), Rothschild (1992), Albæk and Lambertini (1998) and Lambertini and Sasaki $(1999,2002)$, inter alia.
} 
being the smallest discount factors needed for sustaining the monopoly outcome in the repeated game given the market variable(s). In this meta-game firms are supposed to prefer small discount factors. These papers show that a prisoners' dilemma may arise in the meta-game where firms choose the market variable(s), i.e., that the latter may be inefficient.

The present paper nests into the existing literature on the stability of collusion and its relation with product differentiation. We partly rely upon the analysis carried out in Lambertini (1997) and Albæk and Lambertini (2004), with a relevant departure from their line of research. That is, we still suppose that the choice of any given market variable is a long-run commitment to be taken at the outset in the meta-game, but we assume that the relevant payoff in the meta-game are given by discounted profit flows. We shall examine two different setups: one where cartel profits are split evenly between firms, and the other where, in asymmetric (i.e., price-quantity) supergames, the additional profits attainable through collusion are split according to the Nash bargaining solution. These two alternatives have largely different consequences in terms of subgame perfect equilibrium outcomes. In the game with equal split only symmetric equilibria arises, and firms collude along the subgame perfect equilibrium path if and only if both of them are either priceor quantity-setters. The outcome is significantly different if collusive profits are split following a Nash bargaining solution in the asymmetric cases. If so, there exist parameter ranges (where product differentiation as well as firms' discount factors are sufficiently low) in which firms are indeed able to stabilise price-quantity cartels at the pure-strategy subgame perfect equilibria. That is, asymmetric market behaviour, if combined with Nash bargaining over cartel profits, helps firms collude in such a way that, when a priori symmetric firms are unable to sustain either Bertrand or Cournot cartels, they 
may nonetheless activate price-quantity cartels along the frontier of industry profits.

The remainder of the paper is structured as follows. The demand system id laid out in section 2. Section 3 describes the meta-game. the critical discount factors for each possible supergame are listed in section 4 . The analysis of subgame perfect equilibria is in section 5 . Section 6 contains a few concluding remarks.

\section{Setup}

Two firms, labelled $i$ and $j$, supply the market with a single product each. Firm $i$ 's inverse demand function is

$$
p_{i}=1-q_{i}-\gamma q_{j},
$$

where $\gamma \in(0,1]$ denotes the degree of substitutability between the two products. If $\gamma=0$, firms are independent monopolists, therefore we shall exclude this case in the remainder of the analysis.

The direct demand function faced by firm $i$ is:

$$
q_{i}=\frac{1}{1+\gamma}-\frac{1}{1-\gamma^{2}} p_{i}+\frac{\gamma}{1-\gamma^{2}} p_{j}
$$

When instead firm $i$ acts as a quantity-setter while firm $j$ is a price-setter, their respective demand functions are:

$$
\begin{gathered}
p_{i}=1-q_{i}+\gamma\left(p_{j}+\gamma q_{i}-1\right) ; \\
q_{j}=1-p_{j}-\gamma q_{i} .
\end{gathered}
$$


For the sake of simplicity, we assume firms operate at constant returns to scale and, without further loss of generality, we normalise the marginal cost to zero. Accordingly, each firm's profit function corresponds to revenue, $\pi_{i}=p_{i} q_{i}$.

\section{The meta-game}

The concept of extended game is due to Hamilton and Slutsky (1990; HS henceforth). They consider the extension, out of real time, of the basic duopoly game taking place in real time, in order to endogenise firms' choices as to the timing of moves in the market. This yields a two-stage game, where the first stage concerns the timing, and the second describes market subgames. $^{2}$ Their approach can be adopted to investigate the choice of the market variable as well.

To this aim, consider first an extended or meta-game where firms noncooperatively choose the market variable, knowing that the ensuing market competition takes the form of a one-shot game $G^{1}$. We shall adopt here a symbology which largely replicates that in HS (1990, p. 32). Define $\Gamma^{1,1}=(N, \Sigma, \Pi)$ the extended game. The superscript indicates that both the extension and the basic market game are one-shot. The set of players (or firms) is $N=\{i, j\} ; \alpha_{i}$ and $\alpha_{j}$ are the compact and convex intervals of $\mathbb{R}$ representing the actions available to $i$ and $j$ in the basic game. $\Pi$ is the payoff function. Payoffs depend on the actions undertaken in the basic

\footnotetext{
${ }^{2} \mathrm{HS}$ consider (i) an extended game with observable delay, where firms declare the instant at which they will move, without announcing any particular action; and (ii) an extended game with action commitment, where firms must commit to a specific price or quantity level. The meta-game we describe is conceptually similar to their game with observable delay.
} 
(market) game, according to the following functions, $\pi_{i}: \alpha_{i} \times \alpha_{j} \rightarrow \mathbb{R}$ and $\pi_{j}: \alpha_{j} \times \alpha_{i} \rightarrow \mathbb{R}$. The set of market variables from which firms can choose is $V=\{p, q\}$. The set of strategies for player $i$ is $\Sigma_{i}=\{p, q\} \times \Phi_{i}$, where $\Phi_{i}$ is the set of functions mapping $V \times V$ into $\alpha_{i}$ (or $\left.\alpha_{j}\right)$. Let $\pi_{i}^{h k}$ define the one-shot Nash equilibrium profits for firm $i$, when it chooses market variable $h$ and the rival chooses market variable $k$, with $h, k \in\{p, q\}$. The reduced form of the meta-game can be described as in Matrix $1 .^{3}$

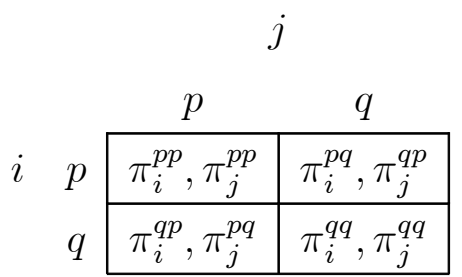

\section{Matrix 1: the one-shot meta-game}

This is the game analysed by Singh and Vives (1984), using the demand functions introduced in the previous section. Since $\pi_{i}^{q p} \geq \pi_{i}^{p p}$ and $\pi_{i}^{q q}>$ $\pi_{i}^{p q}$ for all $\gamma \in(0,1]$, they conclude that firms play the symmetric Cournot equilibrium.

Consider now a meta-game $\Gamma^{1, \infty}$, where the extension is again a oneshot choice over the set $V=\{p, q\}$, but market interaction takes place over an infinite horizon, $t \in[0, \infty)$, giving rise to a supergame $G^{\infty}$. Denote the individual (common) discount factor of players as $\delta \in[0,1] .^{4}$ In this

\footnotetext{
${ }^{3}$ Notice that the extended game envisaged here maintains that firms commit to a strategic market variable for the whole duration of the supergame. Alternatively, one could think of a situation where agents can switch from one variable to the other depending upon the profit incentives characterising each phase of the supergame, i.e., the collusive path, the deviation phase and the punishment. This perspective is considered in Rothschild (1995) and Lambertini and Schultz (2001, 2003).

${ }^{4}$ Although in line of principle players might have different time preferences, the assump-
} 
case, firms can collude in the relevant market variable(s), relying upon an infinite Nash reversion to deter deviations from the collusive path (Friedman, 1971). ${ }^{5}$ That is, we assume that firms either fully collude along the frontier of monopoly profits or play the one-shot Nash equilibrium in the relevant market variable(s). Firms are able to sustain collusion iff

$$
\frac{\pi_{i C}^{h k}}{1-\delta} \geq \pi_{i D}^{h k}+\frac{\delta}{1-\delta} \cdot \pi_{i N}^{h k}
$$

where subscripts $C, D$, and $N$ identify cartel, deviation and Nash profits, respectively. Let $\delta^{h k}$ define the critical discount factor above which firms can indeed collude in the repeated market game; then, the discounted flow of profits accruing to firm $i$ is:

$$
\begin{gathered}
\Psi_{i}^{h k} \equiv \frac{\pi_{i C}^{h k}}{1-\delta} \text { if } \delta \geq \max \left\{\delta^{h k}, \delta^{k h}\right\} \\
\Psi_{i}^{h k} \equiv \frac{\delta}{1-\delta} \cdot \pi_{i N}^{h k} \text { if } \delta \leq \max \left\{\delta^{h k}, \delta^{k h}\right\} .
\end{gathered}
$$

Accordingly, the reduced form of the extended or meta-game $\Gamma^{1, \infty}$ is a $2 \times 2$ matrix analogous to Matrix 1 , with either $\pi_{i C}^{h k} /(1-\delta)$ or $\delta \pi_{i N}^{h k} /(1-\delta)$ replacing $\pi_{i}^{h k}$ as appropriate. The list of admissible outcomes is defined by $\Lambda \equiv\{(p, p),(p, q),(q, p),(q, q)\}$. Given the symmetry of the model, one of the following situations must arise:

- any admissible outcome of the reduced form of $\Gamma^{1, \infty}$ is an equilibrium; tion of common time discounting involves no loss of generality. If we assumed $\delta_{i} \neq \delta_{j}$, then $\min \left\{\delta_{i}, \delta_{j}\right\}$ would determine whether any given cartel is sustainable or not.

${ }^{5}$ In line of principle, the use of optimal punishments à la Abreu (1986) and Fudenberg and Maskin (1986) would be preferable, but this is prevented by the analytical difficulties associated with characterising optimal punishments in asymmetric games with priceand quantity-setting. For supergames with optimal penal codes, see Häckner (1996) and Lambertini and Sasaki (1999, 2002), inter alia. 
- $\Gamma^{1, \infty}$ is a coordination game, with equilibria $(p, p)$ and $(q, q)$;

- $\Gamma^{1, \infty}$ is a chicken game, with equilibria $(p, q)$ and $(q, p)$;

- $\Gamma^{1, \infty}$ has a unique equilibrium, either $(p, p)$ or $(q, q)$. If so, two subcases are possible. Either the unique equilibrium is Pareto-efficient, or not, i.e., the game is a Prisoners' Dilemma.

- no pure-strategy equilibrium exists.

The subgame perfect equilibrium outcome(s) will of course depend upon the value of parameters $\gamma$ and $\delta$.

\section{The critical discount factors}

Consider the market supergame $G^{\infty}$. Since we rely upon "grim" strategies (Friedman, 1971) to model the supergame, we may borrow from Deneckere (1983, 1984) the following discount factors characterising the Cournot and Bertrand settings, respectively:

$$
\begin{gathered}
\delta^{q q}=\frac{(\gamma+2)^{2}}{\gamma^{2}+8 \gamma+8} ; \delta^{p p}=\frac{(\gamma-2)^{2}}{\gamma^{2}-8 \gamma+8} ; \gamma \in(0, \sqrt{3}-1), \\
\delta^{q q}=\frac{(\gamma+2)^{2}}{\gamma^{2}+8 \gamma+8} ; \delta^{p p}=\frac{(2-\gamma)^{2}\left(\gamma^{2}+\gamma-1\right)}{(2-\gamma)^{2}\left(\gamma^{2}+\gamma-1\right)+\gamma^{4}} ; \gamma \in[\sqrt{3}-1,1],
\end{gathered}
$$

where superscript $q q(p p)$ indicates that both firms set quantities (resp., prices). The threshold value $\gamma=\sqrt{3}-1$ identifies the level of substitutability above which deviation from the collusive path drives a loyal price-setting firm out of business.

As $\delta^{q q}<\delta^{p p}$ for all $\gamma \in(0,0.96155)$, Deneckere (1983) concludes that Cournot behavior ensures greater stability of the cartel agreement than does 
Bertrand behavior, unless products are very close substitutes. However, observe that $\delta^{q q}$ is everywhere increasing in $\gamma$, while $\delta^{p p}$ is non-monotone in $\gamma$, with a maximum at $\gamma=2(\sqrt{10}-2) / 3 \simeq 0.774852$. This means that a cartel in quantities becomes less stable as product substitutability increases, while, over most of the relevant range of $\gamma$, the opposite holds for a cartel in prices. As stressed in previous literature (Deneckere, 1983; Lambertini and Sasaki, 1999, 2002), this depends on the fact that the balance between the temptation to deviate and the harshness of the punishment goes in opposite directions in the two cases. The performance of $\delta^{p p}$ is also noteworthy in relation with the corresponding critical threshold emerging from Hotelling models, where the minimum discount factor required to stabilise price collusion increases as product differentiation decreases (see Chang, 1991, 1992; Ross, 1992; Häckner, 1996). This is due to the assumption, usually adopted in Hotelling models, of a price-inelastic market demand, whereby the overall number of consumers being served in each phase of the supergame is independent of the pricing behaviour of firms in that particular phase.

When firms use different market variables, the demand functions are (3) and (4). Straightforward computations yield the following Nash equilibrium profits:

$$
\pi_{i N}^{q p}=\frac{(\gamma-2)^{2}\left(1-\gamma^{2}\right)}{\left(3 \gamma^{2}-4\right)^{2}} ; \pi_{j N}^{p q}=\frac{(\gamma-1)^{2}(\gamma+2)^{2}}{\left(3 \gamma^{2}-4\right)^{2}}
$$

where firm $i$ is a quantity-setter, while firm $j$ is a price-setter. The maximization of joint profits requires $q_{i C}=1 /(2(1+\gamma))$ and $p_{j C}=1 / 2$. This pair of strategies yields total cartel profits equal to monopoly profits, $\pi_{M}=$ $1 /(2(1+\gamma))$ with each firm being entitled to half the cartel profits, i.e., $\pi_{i C}^{q p}=\pi_{j C}^{p q}=\pi_{M} / 2$.

As to the deviation phase, the individually optimal deviation output 
(price) when the other firm sticks to the collusive price (output) corresponds to $q_{i D}=1 /(2 \gamma)$ and $p_{j D}=(2+\gamma) /(4(1+\gamma))$. Notice that $q_{i D}$ coincides with the monopoly output, given only one firm on the market, since the deviation by the quantity setting firm drives the price setting firm completely out of the market. The corresponding deviation profits are: ${ }^{6}$

$$
\pi_{i D}^{q p}=\frac{2 \gamma-1}{4 \gamma^{2}} ; \pi_{j D}^{p q}=\frac{(\gamma+2)^{2}}{16(1+\gamma)^{2}}
$$

yielding the following profits for the firm being cheated:

$$
\bar{\pi}_{j}^{p q}=0 ; \bar{\pi}_{i}^{q p}=\frac{2+2 \gamma-\gamma^{2}}{8(\gamma+1)^{2}}
$$

Since $\pi_{i D}^{q p}>\pi_{i C}^{q p}>\pi_{i N}^{q p}>\bar{\pi}_{i}^{q p}$, and $\pi_{j D}^{p q}>\pi_{j C}^{p q}>\pi_{j N}^{p q}>\bar{\pi}_{j}^{p}$ hold for the quantity-setter and the price-setter, respectively, the mixed situation where firms optimize in different variables reproduces the Prisoners' Dilemma. As in the pure price or quantity games, a Pareto-improvement on the noncooperative outcome can be reached in the repeated game over an infinite horizon, if firms $i$ and $j$ 's discount factors are at least as high as the critical thresholds, defined as:

$$
\delta^{q p}=\frac{\pi_{i D}^{q p}-\pi_{i C}^{q p}}{\pi_{i D}^{q p}-\pi_{i N}^{q p}} ; \delta^{p q}=\frac{\pi_{i D}^{p q}-\pi_{i C}^{p q}}{\pi_{i D}^{p q}-\pi_{i N}^{p q}},
$$

where superscript $q p(p q)$ indicates that the discount factor is computed for the quantity-setter (price-setter), and $\pi_{i C}^{q p}=\pi_{i C}^{p q}=\pi_{M} / 2$. After some simple albeit tedious calculations, one finds that the individual discount factors must satisfy the following inequalities:

$$
\delta_{i} \geq \delta^{q p}=\frac{\left(3 \gamma^{2}-4\right)^{2}}{(\gamma-2)^{2}\left(8-7 \gamma^{2}\right)} ; \gamma \in(0, \sqrt{3}-1),
$$

\footnotetext{
${ }^{6}$ For further details on the calculations concerning the asymmetric cases, we refer to Lambertini (1997) and Albæk and Lambertini (2004).
} 


$$
\begin{gathered}
\delta_{i} \geq \delta^{q p}=\frac{\left(3 \gamma^{2}-4\right)^{2}\left(\gamma^{2}+\gamma-1\right)}{\left(4 \gamma^{7}+6 \gamma^{6}+5 \gamma^{5}-29 \gamma^{4}-24 \gamma^{3}+40 \gamma^{2}+16 \gamma-16\right)} ; \gamma \in[\sqrt{3}-1,1] . \\
\delta_{j} \geq \delta^{p q}=\frac{\left(3 \gamma^{2}-4\right)^{2}}{(\gamma+2)^{2}\left(8-7 \gamma^{2}\right)} ; \gamma \in(0, \sqrt{3}-1) \\
\delta_{j} \geq \delta^{p q}=\frac{\left(3 \gamma^{2}-4\right)^{2}}{(\gamma+2)^{2}\left(8-7 \gamma^{2}\right)} ; \gamma \in[\sqrt{3}-1,1] .
\end{gathered}
$$

Alternatively, in the price-quantity case, firms could split evenly the additional profits made available by collusion, in the light of the asymmetry between non-cooperative profits:

$$
\widehat{\pi}_{i C}^{q p}=\pi_{i N}^{q p}+\frac{\pi_{M}-\pi_{i N}^{q p}-\pi_{j N}^{p q}}{2} ; \widehat{\pi}_{i C}^{p q}=\pi_{i N}^{p q}+\frac{\pi_{M}-\pi_{i N}^{q p}-\pi_{j N}^{p q}}{2}
$$

where:

$$
\widehat{\pi}_{i C}^{q p}>\pi_{i C}^{q p} ; \widehat{\pi}_{j C}^{p q}<\pi_{j C}^{p q} \quad \forall \gamma \in(0,1] .
$$

If rule (14) is adopted, the critical discount factors are defined as follows:

$$
\widehat{\delta}^{q p}=\frac{\pi_{i D}^{q p}-\widehat{\pi}_{i C}^{q p}}{\pi_{i D}^{q p}-\pi_{i N}^{q p}} ; \widehat{\delta}^{p q}=\frac{\pi_{i D}^{p q}-\widehat{\pi}_{i C}^{p q}}{\pi_{i D}^{p q}-\pi_{i N}^{p q}} .
$$

Again, routine calculations are required to find that the individual discount factors must satisfy the following inequalities:

$$
\begin{gathered}
\delta_{i}>\widehat{\delta}^{q p}=\frac{16-16 \gamma-8 \gamma^{2}+16 \gamma^{3}-7 \gamma^{4}}{(\gamma-2)^{2}\left(8-7 \gamma^{2}\right)} ; \gamma \in(0, \sqrt{3}-1) \\
\delta_{i} \geq \widehat{\delta}^{q p}=\frac{4 \gamma^{7}+9 \gamma^{6}+5 \gamma^{5}-33 \gamma^{4}-24 \gamma^{3}+40 \gamma^{2}+16 \gamma-16}{4 \gamma^{7}+6 \gamma^{6}+5 \gamma^{5}-29 \gamma^{4}-24 \gamma^{3}+40 \gamma^{2}+16 \gamma-16} ; \gamma \in[\sqrt{3}-1,1] . \\
\delta_{j}>\widehat{\delta}^{p q}=\frac{16+16 \gamma-8 \gamma^{2}-16 \gamma^{3}-7 \gamma^{4}}{(\gamma+2)^{2}\left(8-7 \gamma^{2}\right)} ; \gamma \in(0, \sqrt{3}-1)
\end{gathered}
$$




$$
\delta_{j} \geq \widehat{\delta}^{p q}=\frac{16+16 \gamma-8 \gamma^{2}-16 \gamma^{3}-7 \gamma^{4}}{(\gamma+2)^{2}\left(8-7 \gamma^{2}\right)} ; \gamma \in[\sqrt{3}-1,1] .
$$

Having derived the critical threshold of discount factors for each supergame, we may proceed to investigate the upstream stage of the game.

\section{The upstream stage}

We are now in a position to fully characterise the subgame perfect equilibrium behaviour of firms in the game $\Gamma^{1, \infty}$. The normal form of the upstream stage of the game is described by Matrix 2:

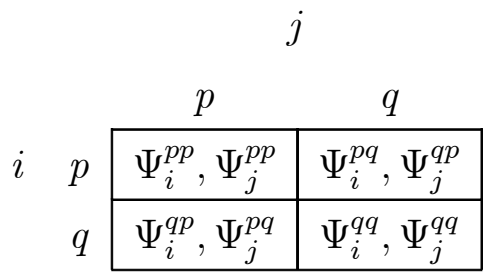

\section{Matrix 2}

where $\Psi_{i}^{h k}$ is given by either $\pi_{i C}^{h k} /(1-\delta)$ or $\delta \pi_{i N}^{h k} /(1-\delta)$, depending on $\delta \gtreqless \max \left\{\delta^{h k}, \delta^{k h}\right\}$. We first investigate the case where firms split cartel profits evenly.

\subsection{The game with equal split}

A first result immediately obtains with bo need of a formal proof:

Lemma 1 If $\delta \geq \max \left\{\delta^{q p}, \delta^{q q}, \delta^{p p}, \delta^{p q}\right\}$, firms are always able to stabilise collusion, irrespective of the market variable(s) being chosen. Otherwise, if 
$\delta<\min \left\{\delta^{q p}, \delta^{q q}, \delta^{p p}, \delta^{p q}\right\}$, firms are completely unable to collude. In such a case, non-cooperative quantity-setting is a dominant strategy and firms play à la Cournot-Nash forever.

The first claim in the Lemma also entails that, if $\delta$ is sufficiently high, the choice of the market variable is indeed immaterial to firms, since they always obtain their share of monopoly profits. The second claim summarises the main result obtained by Singh and Vives (1984).

Now examine all the intermediate cases where

$$
\delta \in\left[\min \left\{\delta^{q p}, \delta^{q q}, \delta^{p p}, \delta^{p q}\right\}, \max \left\{\delta^{q p}, \delta^{q q}, \delta^{p p}, \delta^{p q}\right\}\right) .
$$

The relevant sequence of the critical discount factors is:

$$
\begin{aligned}
& \delta^{q p}>\delta^{p p}>\delta^{q q}>\delta^{p q} \text { for all } \gamma \in(0,0.96155), \\
& \delta^{q p}>\delta^{q q} \geq \delta^{p p}>\delta^{p q} \text { for all } \gamma \in[0.96155,1] .
\end{aligned}
$$

Suppose $\gamma \in(0,0.96155)$, and:

$$
\delta^{q p}>\delta \geq \delta^{p p}>\delta^{q q}>\delta^{p q}
$$

If so, the relevant matrix is:

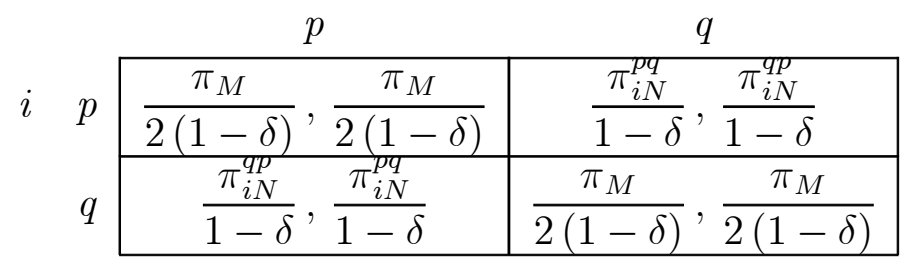

\section{Matrix 3}

Matrix 3 represents a coordination game whose pure-strategy equilibria are $(p, p)$ and $(q, q)$, with firms colluding symmetrically, either in prices or 
in output levels. Of course, there also exist a mixed-strategy equilibrium as well as a correlated one.

Again for $\gamma \in(0,0.96155)$, suppose:

$$
\delta^{q p}>\delta^{p p}>\delta \geq \delta^{q q}>\delta^{p q} .
$$

The reduced-form matrix becomes:

$$
\begin{aligned}
& \text { j }
\end{aligned}
$$

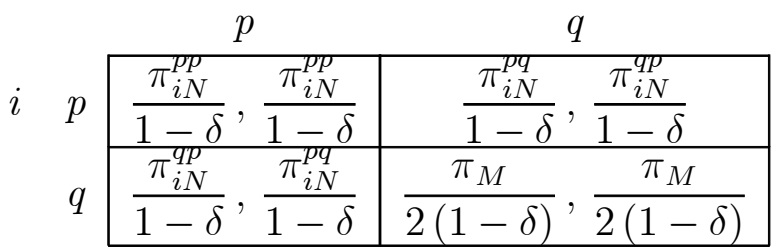

\section{Matrix 4}

In this case, firms collude only if both set quantities, and the game produces a unique equilibrium in dominant strategies, $(q, q)$, which is also Pareto-efficient for firms.

If $\delta^{q p}>\delta^{p p}>\delta \geq \delta^{q q}>\delta \geq \delta^{p q}$, firms are unable to collude irrespective of the market variables being chosen, and the equilibrium outcome is the same as in Singh and Vives (1984), with $(q, q)$ representing the unique equilibrium (in dominant strategies), which is also Pareto-efficient.

Now suppose $\gamma \in[0.96155,1]$. To begin with, examine the case where:

$$
\delta^{q p}>\delta \geq \delta^{q q} \geq \delta^{p p}>\delta^{p q}
$$

This situation is qualitatively analogous to that described in Matrix 3, so that there are two pure-strategy equilibria, $(p, p)$ and $(q, q)$, where firms can sustain collusion. However, in mixed strategies they can be driven off the collusive path and play one-shot asymmetric Nash equilibria. 
If instead:

$$
\delta^{q p}>\delta \geq \delta^{q q} \geq \delta \geq \delta^{p p}>\delta^{p q},
$$

the relevant matrix is:

$$
\begin{aligned}
& \text { j }
\end{aligned}
$$

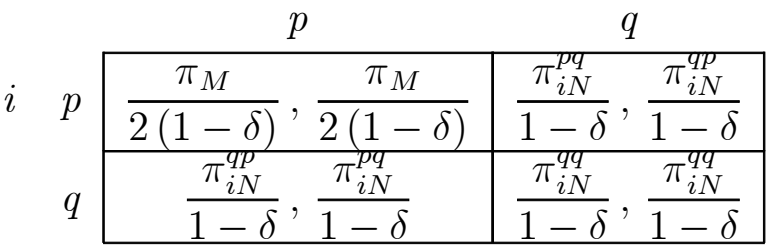

\section{Matrix 5}

This game has also two equilibria in pure strategies, $(p, p)$ and $(q, q)$, but firms collude if and only if the are price-setters. Finally, if $\delta^{q p}>\delta \geq \delta^{q q} \geq$ $\delta^{p p}>\delta \geq \delta^{p q}$, firms never collude, ending up playing a Cournot-Nash equilibrium forever.

The foregoing discussion is summarised by:

Proposition 2 In the game with equal split, if $\delta<\max \left\{\delta^{q p}, \delta^{q q}, \delta^{p p}, \delta^{p q}\right\}$, then only symmetric equilibria may arise. Price-quantity supergames are never part of the subgame perfect equilibrium path.

The relative stability of Cournot and Bertrand implicit cartels is nonmonotone w.r.t. the degree of product substitutability, as it is well known from previous literature and further stressed here, e.g., by comparing Matrices 4 and 5 . However, the relative profitability of price and quantity behaviour is such that collusion between a price- and a quantity-setter is never to be observed along the subgame perfect equilibrium path. 


\subsection{The game with Nash bargaining}

Here we consider the situation where firms split additional collusive profits as in (14), so that the relevant critical discount factors characterising the asymmetric market supergames where one firm is a price-setter and the other is a quantity-setters are given by (16). The equivalent of Lemma 1 also holds here.

Then, although $\delta^{q p}>\widehat{\delta}^{q p}$ and $\widehat{\delta}^{p q}>\delta^{p q}$, the sequence of critical discount factors remains the same as under equal split:

$$
\widehat{\delta}^{q p}>\delta^{p p}>\delta^{q q}>\widehat{\delta}^{p q}
$$

for all $\gamma \in(0, \sqrt{3}-1)$. In this range, the upstream stage of the game $\Gamma^{1, \infty}$ has exactly the same qualitative features as in the game with equal split. Therefore, firms may collude only if they use the same market variable.

Things modify significantly when $\gamma \in[\sqrt{3}-1,1]$. In this parameter region, the following sequences hold:

$$
\begin{gathered}
\widehat{\delta}^{q p}>\delta^{p p}>\delta^{q q}>\widehat{\delta}^{p q} \text { for all } \gamma \in[\sqrt{3}-1,0.87311), \\
\delta^{p p} \geq \widehat{\delta}^{q p}>\delta^{q q}>\widehat{\delta}^{p q} \text { for all } \gamma \in[0.87311,0.93600), \\
\delta^{p p}>\delta^{q q} \geq \widehat{\delta}^{q p}>\widehat{\delta}^{p q} \text { for all } \gamma \in[0.93600,0.96155), \\
\delta^{q q} \geq \delta^{p p}>\widehat{\delta}^{q p}>\widehat{\delta}^{p q} \text { for all } \gamma \in[0.96155,1] .
\end{gathered}
$$

When $\gamma \in[\sqrt{3}-1,0.87311)$ so that (26) holds, and:

$$
\widehat{\delta}^{q p}>\delta \geq \delta^{p p}>\delta^{q q}>\widehat{\delta}^{p q}
$$

we have a coordination game, as in Matrix 3. If instead:

$$
\widehat{\delta}^{q p}>\delta^{p p}>\delta \geq \delta^{q q}>\widehat{\delta}^{p q}
$$


$(q, q)$ is the unique (collusive) equilibrium in dominant strategies, as in Matrix 4. Otherwise, if $\delta \in\left(\delta^{q q}, \widehat{\delta}^{p q}\right]$ firms are unable to collude and play the Cournot-Nash equilibrium forever.

Now suppose $\gamma \in[0.87311,0.93600)$, with the relevant sequence being (27). If

$$
\delta^{p p} \geq \delta \geq \widehat{\delta}^{q p}>\delta^{q q}>\widehat{\delta}^{p q}
$$

both firms choose strategy $q$ and collude. they are able to collude also when one sets the price and the other sets the output level, but, by dominance, a Cournot cartel emerges as the unique equilibrium. The same equilibrium outcome also obtains if $\delta^{p p} \geq \widehat{\delta}^{q p}>\delta \geq \delta^{q q}>\widehat{\delta}^{p q}$, where the reduced form appears as in Matrix 4. If $\delta$ is even lower, than firms play à la Cournot-Nash.

Then, consider $\gamma \in[0.93600,0.96155)$ and (28). If $\delta^{p p}>\delta \geq \delta^{q q} \geq \widehat{\delta}^{q p}>$ $\widehat{\delta}^{p q}$, firms always collude except in the Bertrand supergame. Dominance establishes that $(q, q)$ is the unique (collusive) equilibrium. If instead $\delta^{p p}>$ $\delta^{q q} \geq \delta \geq \widehat{\delta}^{q p}>\widehat{\delta}^{p q}$, the reduced form of the upstream stage is:

\begin{tabular}{|c|c|c|}
\hline \multirow[b]{3}{*}{$p$} & $p$ & $q$ \\
\hline & $\pi_{i N}^{p p} \quad \pi_{i N}^{p p}$ & $\widehat{\pi}_{i C}^{p q} \quad \widehat{\pi}_{i C}^{q p}$ \\
\hline & $\overline{1-\delta}, \overline{1-\delta}$ & $\overline{1-\delta}, \overline{1-\delta}$ \\
\hline$q$ & $\frac{\widehat{\pi}_{i C}^{q p}}{1-\delta}, \frac{\widehat{\pi}_{i C}^{p q}}{1-\delta}$ & $\frac{\pi_{i N}^{q \varphi}}{1-\delta}, \frac{\pi_{i N}^{q \varphi}}{1-\delta}$ \\
\hline
\end{tabular}

Matrix 6

Since $\widehat{\pi}_{i C}^{q p}>\pi_{i N}^{p p}$ and $\widehat{\pi}_{i C}^{q p}>\pi_{i N}^{q q}$, Matrix 6 describes a chicken game with two pure strategy equilibria where firms collude, $(p, q)$ and $(q, p)$, plus of course a mixed strategy equilibrium and a correlated one. If $\delta \in\left(\widehat{\delta}^{q p}, \widehat{\delta}^{p q}\right]$, Singh and Vives's (1984) result applies. 
Finally, if $\gamma \in[0.96155,1]$ and sequence $(29)$ holds, then the reduced form of the upstream stage is a chicken game if either $\delta \in\left(\delta^{q q}, \delta^{p p}\right]$, since $\widehat{\pi}_{i C}^{q p}>\pi_{M} / 2$, or $\delta \in\left(\delta^{p p}, \widehat{\delta}^{q p}\right]$, where firms play à la Cournot- and BertrandNash along the main diagonal, while they collude otherwise.

The analysis carried out above is summarised by:

Proposition 3 In the game with Nash bargaining, if $\delta<\max \left\{\widehat{\delta}^{q p}, \delta^{q q}, \delta^{p p}, \widehat{\delta}^{p q}\right\}$, any admissible outcome can be an equilibrium, depending upon the value of parameters $\delta$ and $\gamma$. In particular, if products are sufficiently close substitutes and firms' discount factor takes intermediate values, the game produces two asymmetric equilibria in pure strategies, with firms playing a collusive price-quantity supergame.

The reason for this result is to be found in the fact that Nash bargaining over the cartel profits, by increasing (respectively, decreasing) the collusive profits accruing to the quantity-setter (resp., price-setter) indeed makes collusion in price-quantity settings easier to sustain than it is under equal split.

\section{Concluding remarks}

We have revisited the long-standing debate about the stability of collusion in differentiated duopolies, by nesting the supergame in an extended game with observable delay, in which firms non-cooperatively select the market variable in a pre-play stage, in view of the discounted profit flows generated by the ensuing market supergame.

The analysis of the game where cartel profits are evenly split between firms has shown that collusion is part of the subgame perfect equilibrium if and only if both firms are either price- or quantity-setters. Conclusions may 
be drastically different if collusive profits are distributed between firms using a Nash bargaining solution. In such a case, when product differentiation is relatively low and firms are sufficiently myopic, the sustainability of symmetric cartels becomes too demanding and there emerge pure-strategy equilibria where firms stabilise price-quantity cartels. Therefore, it appears that asymmetric market behaviour, coupled with Nash bargaining over the additional cartel profits made available through collusion, can help firms build up stable cartels. This is an additional reason for antitrust agencies to worry about implicit collusion, as it adds up to the established theoretical wisdom on the matter a further source of collusive behaviour that previous literature had either neglected or simply regarded as a lesser evil. Indeed, this may be due to the idea that an a priori symmetric duopoly should generate, if at all, symmetric cartels. the foregoing analysis shows that there are circumstances where this may not be the case.

\section{References}

[1] Abreu, D.J. (1986), Extremal Equilibria of Oligopolistic Supergames, Journal of Economic Theory, 39, 191-225.

[2] Albæk, S. and L. Lambertini (1998), Collusion in Differentiated Duopolies Revisited, Economics Letters, 59, 305-308.

[3] Albæk, S. and Lambertini L. (2004), Price vs Quantity in Duopoly Supergames with Close Substitues, Annals of Regional Science, forthcoming.

[4] Chang, M.H. (1991), The Effects of Product Differentiation on Collusive Pricing, International Journal of Industrial Organization, 9, 453-469. 
[5] Chang, M.H. (1992), Intertemporal Product Choice and Its Effects on Collusive Firm Behavior, International Economic Review, 33, 773-793.

[6] Deneckere, R. (1983), Duopoly Supergames with Product Differentation, Economics Letters, 11, 37-42.

[7] Deneckere, R. (1984), Corrigenda, Economics Letters, 15, 385-387.

[8] Friedman, J.W. (1971), A Non-Cooperative Equilibrium for Supergames, Review of Economic Studies, 28, 1-12.

[9] Fudenberg, D, and E. Maskin (1986), The Folk Theorem in Repeated Games with Discounting or with Incomplete Information, Econometrica, 54, 533-554.

[10] Häckner, J. (1996), Optimal Symmetric Punishments in a Bertrand Differentiated Product Duopoly, International Journal of Industrial Organization, 14, 611-630.

[11] Hamilton, J. and S. Slutsky (1990), Endogenous Timing in Duopoly Games: Stackelberg or Cournot Equilibria, Games and Economic Behavior, 2, 29-47.

[12] Lambertini, L. (1997), Prisoners' Dilemma in Duopoly (Super)Games, Journal of Economic Theory, 77, 181-191.

[13] Lambertini, L. and C. Schultz (2001), Price vs. Quantity in a Repeated Differentiated Duopoly, working paper no. 379, Department of Economics, University of Bologna.

[14] Lambertini, L. and C. Schultz (2003), Price or Quantity in Tacit Collusion?, Economics Letters, 78, 131-137. 
[15] Lambertini, L. and D. Sasaki (1999), Optimal Punishments in Linear Duopoly Supergames with Product Differentation, Journal of Economics, 69, 173-188.

[16] Lambertini, L. and D. Sasaky (2002), Non-Negativity Quantity Constraints and the Duration of Punishment, Japanese Economic Review, 53, 77-93.

[17] Majerus, D. (1988), Price vs. Quantity Competition in Oligopoly Supergames, Economics Letters, 27, 293-297.

[18] Ross, T.W. (1992), Cartel Stability and Product Differentiation, International Journal of Industrial Organization, 10, 1-13.

[19] Rothschild, R. (1992), On the Sustainability of Collusion in Differentiated Duopolies, Economics Letters, 40, 33-37.

[20] Rothschild, R. (1995), Sustaining Collusion when the Choice of Strategic Variable Is Endogenous, Journal of Economic Behavior and Organization, 28, 373-385.

[21] Singh, N. and X. Vives (1984), Price and Quantity Competition in a Differentiated Duopoly, Rand Journal of Economics, 15, 546-554. 